\title{
Energy harvesting of magnetic power-line noise
}

\author{
Kunihisa Tashiro ${ }^{1}$, Hiroyuki Wakiwaka ${ }^{1}$, Shin-ichiro Inoue ${ }^{1}$ and Yu Uchiyama ${ }^{1}$ \\ ${ }^{1}$ Spin device technology center, Shinshu University, Nagano, Wakasato 4-17-1, Japan
}

\begin{abstract}
The purpose of this study is to demonstrate the energy harvesting of power line magnetic noise. To the best of the authors' knowledge, no research has yet been carried out to confirm this possibility. In this paper, we present a simple circuit design model, and confirm its validity by experimental results. For small self-sufficient devices in a wireless sensor network, the target energy level is 1 $\mathrm{mW}$. We prepare an energy harvesting module with an air-core coil and resonant capacitor. From experimental and simulated results, we investigate the desired conditions for harvesting $1 \mathrm{~mW}$ from a uniform magnetic field of $60 \mathrm{~Hz}$. Through experimental results with iron cores, the benefits and drawbacks of the use of a magnetic core are also discussed. With our best harvested module, we successfully demonstrated the energy harvesting of $6.32 \mathrm{~mW}$ from a magnetic field of $21.2 \mu \mathrm{T}$ at $60 \mathrm{~Hz}$. If the usable magnetic flux density increases 10-fold, the harvesting energy increased 100-fold. If the magnetic flux density is at an acceptable level in a public space of $200 \mu \mathrm{T}$ at $60 \mathrm{~Hz}$, the harvesting power density becomes $130 \mu \mathrm{W} / \mathrm{cm}^{3}$. This value is comparable to the value of an energy harvesting module of a light source during a cloudy day.
\end{abstract}

Index Terms—Energy harvesting, power line, magnetic noise, Brooks coil

\section{INTRODUCTION}

$\mathrm{W}^{\mathrm{s}}$ E can easily find the existence of light, vibration, and thermal energy in our living environment. For harvesting this kind of energy with high efficiency, a number of energy harvesting modules have been developed in recent years. Energy harvesting is a key technology for small selfsufficient devices, which use environmental energy as a source. If the target of the devices is a wireless sensor network, the desired energy is a few $\mathrm{mW}$.

An interested summary is reported by Roundy's group [1]. According to the report, the typical power density is 15,000 $\mu \mathrm{W} / \mathrm{cm}^{3}$ of light energy (outdoor, direct sun), $250 \mu \mathrm{W} / \mathrm{cm}^{3}$ of vibration energy (Piezoelectric conversion), and $15 \mu \mathrm{W} / \mathrm{cm}^{3}$ of thermal energy (at $10{ }^{\circ} \mathrm{C}$ gradient). Compared with batteries and fuel cells, these values are remarkable. For example, a non-rechargeable lithium battery is a promising energy storage device. However, the power density is $45 \mu \mathrm{W} / \mathrm{cm}^{3}$ for a one year lifetime, and $3.5 \mu \mathrm{W} / \mathrm{cm}^{3}$ for a ten year lifetime. Of course, an exact comparison is not easy because their abilities depend on different parameters. When using a harvesting module for light energy sources, the energy density is dramatically decreased to $150 \mu \mathrm{W} / \mathrm{cm}^{3}$ during a cloudy day, and $6 \mu \mathrm{W} / \mathrm{cm}^{3}$ at an office desk.

From the view point of an engineer concerned with weak and low-frequency magnetic field detection, the magnetic fields are considered harmful noise. The authors' group has developed an air-cored induction magnetometer [2]-[6]. According to the circuit simulator, although its sensitivity is comparable to a SQUID sensor we did not successfully observe the waveform of a weak magnetic field of less than 1 pT in our laboratory environment. Noise always exists in our living environment, and a perfect magnetic shielding is not practical.

Because power-lines are necessary for our modern life, we are required to coexist with undesirable magnetic power-line

Manuscript received February 21, 2011. Corresponding author: Kunihisa Tashiro (e-mail: tashiro@shinshu-u.ac.jp).

Digital Object Identifier inserted by IEEE noise. From the view point of the effect on humans, an acceptable level in a public space is $200 \mu \mathrm{T}$ at power-line frequency $(50 \mathrm{~Hz}$ or $60 \mathrm{~Hz})$ [7]. We focus on the magnetic field as a source of energy harvesting. The purpose of this study is to demonstrate energy harvesting of power line magnetic noise. To the best of the authors' knowledge, no research has yet been carried out to confirm this possibility. In this paper, we present the circuit design of a simple model, and confirm the validity with experimental results.

\section{MethoD}

We chose a Brooks coil for the shape of the air-core coil. A Brooks coil is a circular coil having a square cross section, and the ratio of the coil length, inner diameter, and outer diameter is $1: 2: 4$ [8]. In order to estimate the inductance of an air-core coil to three significant digits, we should choose a suitable equation from five equations with four parameters (inner diameter, outer diameter, coil width, and number of windings) [8]. In contrast, the required number of parameters for a Brooks coil is only two, and the estimation error due to the spacing factor [3] and tolerance [4] is quite low. Because this coil shape can achieve the maximum inductance for a given length of winding wire, it is suitable for development of a resonance circuit.

Fig.1 shows our prepared energy harvesting modules, and Table 1 shows the specifications. The values of the inductance $L(\mathrm{H})$ and resistance $R(\Omega)$ were estimated as

$$
\begin{aligned}
& L=P_{0} a n^{2}(\mathrm{H}), \\
& R=2 \pi a n \rho / s(\Omega) .
\end{aligned}
$$

Where, $P_{0}=1.6994 \times 10^{-6}(\mathrm{H} / \mathrm{m})$ is the coil constant for the Brooks coil, $a(\mathrm{~m})$ is the mean radius, $n$ is the number of windings, $\rho(\Omega \bullet \mathrm{m})$ is the resistivity of copper at room temperature, and $s\left(\mathrm{~m}^{2}\right)$ is the cross section area of the winding. The measured values were in good agreement with the estimated values within $\pm 3 \%$ relative error. To provide a resonance frequency at $60 \mathrm{~Hz}$, we chose the resonant capacitor $C$ (F) from 


$$
f=1 / 2 \pi(L C)^{1 / 2}(\mathrm{~Hz}) .
$$

From the maximum power transfer theorem, we chose a load $R_{\mathrm{L}}(\Omega)$ to be the same as the coil resistance and used the voltage drop as the output voltage $V_{\text {out }}(\mathrm{V})$ for calculating the harvesting energy $W(\mathrm{~W})$ with

$$
W=V_{\text {out }}{ }^{2} / R(\mathrm{~W}) .
$$

Fig. 2 shows a model for an energy harvesting module for a circuit simulator (LTSpice, LinearTechnology Inc.). From Faraday's law of induction and Thévenin's theorem, the amplitude of the voltage source $V_{\text {in }}(\mathrm{V})$ can be expressed by

$$
V_{\text {in }}=2 \pi^{2} f n a^{2} B(\mathrm{~V}) .
$$

Where, $f(\mathrm{~Hz})$ is the frequency and $B(\mathrm{~T})$ is the mean flux density crossed with the mean cross section of the coil. If the spacing factor and mean radius of the coil are high, the amplitude of the voltage source and the quality factor are high. However, the energy loss due to the coil resistance becomes high and undesired eddy current losses in the coil may also affect the harvesting energy. From these reasons, we prepared four coils with different radii and spacing factors.

For generating a uniform magnetic field, we used our developed Simple-Cubic-3 coil system (SC3). SC3 was composed of three square coils connected in series, and the distance was the half side length of the coil. The side length was $2 \mathrm{~m}$, and the number of windings was 24:12:24. In the area within a radius of $50 \mathrm{~cm}$ from the center, SC3 can generate a uniform magnetic field within $\pm 1 \%$ deviation. Because the maximum diameter of the modules was $28 \mathrm{~cm}$, we used this area to simulate power line magnetic noise. For the evaluation of frequency dependence, the output voltage was measured with an FFT analyzer. The frequency range was from $1 \mathrm{~Hz}$ to $1 \mathrm{kHz}$, and the amplitude was $1 \mu \mathrm{T}_{\mathrm{p}-\mathrm{p}}$ or $10 \mu \mathrm{T}_{\mathrm{p}-\mathrm{p}}$. For the evaluation of amplitude dependence, the harvesting energy was calculated from the measured output voltage. The frequency was $60 \mathrm{~Hz}$, and the amplitude ranges were from 1 $\mu \mathrm{T}$ to $10 \mu \mathrm{T}_{\mathrm{p}-\mathrm{p}}$ with a $1 \mu \mathrm{T}$ step and from $10 \mu \mathrm{T}_{\mathrm{p}-\mathrm{p}}$ to $60 \mu \mathrm{T}_{\mathrm{p}-\mathrm{p}}$ with a $10 \mu \mathrm{T}_{\mathrm{p}-\mathrm{p}}$ step. The maximum amplitude of the magnetic flux density of $60 \mu \mathrm{T}_{\mathrm{p}-\mathrm{p}}$ corresponds to $21.2 \mu \mathrm{T}(\mathrm{rms})$.

In order to discuss the benefits and drawbacks of the use of a magnetic core, we prepared iron rods whose diameter and length were $25 \mathrm{~mm}$ and $105 \mathrm{~mm}$, respectively (Niraco. Inc., Fe $99.5 \%$ ). The tested number of rods was 1, 2, 3, 4, and 7. They were placed inside the Coill parallel to the center axis. We selected resonant capacitors from the measured results of the inductance using an impedance analyzer. Table 2 shows the measured inductance and the selected resonant capacitors. The amplitude dependency was also measured as mentioned before, and the increasing rates of the inductance and harvested energy were compared.

\section{ResUlts}

Fig. 3 shows examples of the frequency dependency of the modules. Although the modules Coil1 and Coil2 showed a clear peak voltage at $60 \mathrm{~Hz}$, the modules Coil3 and Coil4 did not because the half band widths were larger than $60 \mathrm{~Hz}$. We thus concluded that the quality factor should be larger than 5 for collecting the energy of a $60 \mathrm{~Hz}$ magnetic field selectively. Compared with the simulated results for a magnetic field of 10 $\mu \mathrm{T}$ at $60 \mathrm{~Hz}$, the relative error values for Coill, 2, 3, and 4 were $-40 \%,-42 \%,-48 \%$ and $+9 \%$, respectively.

Fig. 4 shows the amplitude dependency of the modules. Plots represent the measured results, and lines represent the simulated results. Compared with the simulated results, the average values of relative error for Coil1, 2, 3, and 4 were $67 \%,-68 \%,-74 \%$ and $+17 \%$, respectively. It was found that large size and a high spacing factor of the coil made the harvesting energy high. For example the best module, Coil2, harvested $6.32 \mathrm{~mW}$ from a magnetic field of $21.2 \mu \mathrm{T}$ at $60 \mathrm{~Hz}$. Because there were no non-linear materials in the modules, the harvested energy was proportional to the square of the magnetic flux density.

Fig. 5 shows the amplitude dependency of the module, Coill, with iron rods. Plots represent the measured results, and lines represent the simulated results of the module without magnetic material. Without a magnetic core, the module harvested $1.27 \mathrm{~mW}$ from a magnetic field of $21.2 \mu \mathrm{T}$. While the number of inserted iron rods was increased up to 7 , the values of the harvested energy were not remarkable. When we confirmed the amplitude dependency of the modules with or without iron cores, they had the resonant frequency around 60 Hz. However, the peak was not sharp due to the low quality factor. In other word, the imperfection of the resonant capacitor did not affect to the harvested energy, strongly.

Fig. 6 shows the comparison of the normalized values of the inductance and harvested energy. Where, $L_{0}(\mathrm{H})$ and $L(\mathrm{H})$ represent the inductance of the coil, Coil1, without or with iron cores, respectively. $W_{0}(\mathrm{~W})$ and $W(\mathrm{~W})$ represent the harvested energy from a magnetic field of $21.2 \mu \mathrm{T}$ at $60 \mathrm{~Hz}$ with the module, Coill, without or with iron cores. Increasing the number of iron rods produced an increase of the ratio $L / L_{0}$. The normalized inductance values, $100 \times\left(L-L_{0}\right) / L_{0}$, were not dramatic because the effective permeability was limited by the demagnetizing field in the iron rods. It is defined as the ratio of the total length to the diameter. Because the ratio $L / L_{0}$ corresponds to the increasing ratio of the mean flux linkage, the increasing rate of the harvested energy should be proportional to the ratio. However, when the number of inserted iron rods was increased to $1,2,3,4$, and 7 , the harvested energy changed by $-13 \%, 20 \%, 16 \%, 25 \%$, and $26 \%$, respectively. The results indicated the existence of energy loss in the magnetic material. We also calculated the normalized value for the amplitude from $0.35 \mu \mathrm{T}$ to $21.2 \mu \mathrm{T}$. The average values were changed by $-13 \%, 20 \%, 15 \%$, $24 \%$, and $27 \%$, respectively. We concluded that the nonlinearity of the iron rod was not strong in this amplitude range.

\section{Discussion}

Electromagnetic fields are used as an energy medium in an energy harvesting module with a vibration source and wireless power transmission. In contrast, a wireless power transfer method proposed by an MIT group (Magnetic resonant coupling type) [11] has attracted many engineers. However, no research has yet been carried out to harvest the energy of a 
power line magnetic field. In other words, the power line and the noise were not regarded as an energy transmitter and artificial energy source in our living environment.

Table 3 shows the comparison of energy modules. The top and middle parts of the table were summarized by the Roundy group [1]. The bottom part of the table is a summary of our results. The power density was estimated for a one year lifetime. With our best harvesting module, Coil2, we successfully demonstrated energy harvesting of $6.32 \mathrm{~mW}$ from a magnetic field of $21.2 \mu \mathrm{T}$ at $60 \mathrm{~Hz}$. The calculated energy density was $1.47 \mu \mathrm{W} / \mathrm{cm}^{3}$. If the magnetic flux density is an acceptable level in a public space of $200 \mu \mathrm{T}$ at $60 \mathrm{~Hz}$, the estimated harvesting power density becomes $130 \mu \mathrm{W} / \mathrm{cm}^{3}$. This value is comparable to the value of an energy harvesting module of a light source during a cloudy day.

Our experimental results also revealed the problems in the magnetic material. It is not only the effective permeability due to the demagnetizing field, but also the iron loss in the magnetic material. For a reduction of the element size, a suitable magnetic core should be designed. For a preliminary study of the use of magnetic cores, we chose pure iron rods. In order to reduce eddy current loss, laminated electrical steels or amorphous ribbons may also be suitable as core materials.

\section{CONCLUSION}

We developed an energy harvesting module with an air-core coil and a resonant capacitor. With our best harvesting module, we successfully demonstrated the energy harvesting of 6.32 $\mathrm{mW}$ from a magnetic field of $21.2 \mu \mathrm{T}$ at $60 \mathrm{~Hz}$. Although we assumed that magnetic power-line noise is a uniform magnetic field, it may be a very specific case. Power-lines are usually three phase systems where the magnetic field is elliptically polarized. Moreover, the field near the power-lines also has a significant spatial gradient. For practical use, we should also consider these conditions.

\section{ACKNOWLEDGMENT}

This work was supported in part by the Japanese Ministry of Education, Science, Sports and Culture, Grant-in-Aid for Young Scientists (B), under Grant 22760206, 2010-2011.

\section{REFERENCES}

[1] S. Roundy, P. K. Wright, and J. Rabaey, "A study of low level vibrations as a power source for wireless sensor nodes," Computer Communications, vol. 26, pp. 1131-1144, Jul. 2003.

[2] K. Tashiro, "Optimal Design of an Air-Core Induction Magnetometer for Detecting Low-Frequency Fields of Less than $1 \mathrm{pT}$," J. Magn. Soc. Jpn., vol. 30, pp. 439-442, 2006.

[3] K. Tashiro, "Proposal of coil structure for air-core induction magnetometer," Proc. IEEE Sensor 2006, pp. 939- 942, 2006.

[4] K. Tashiro, H. Wakiwaka, A. Kakiuchi, and A. Matsuoka, "Comparative study of air-core coil design for induction magnetometer with current-tovoltage converter," Proc. of second international conference on sensing technology (ICST2007), pp. 590-594, 2007.

[5] K. Tashiro, A. Kakiuchi, K. Moriizumi, and H. Wakiwaka, "An Experimental Study of Stable Operating Conditions for a HighSensitivity Induction Gradiometer," IEEE Trans. on Magn., vol. 45, pp. 2784-2787, Jun 2009.
[6] K. Tashiro, S. Inoue, and H. Wakiwaka, "Sensitivity limits of a magnetometer with an air-core pickup coil," Sensors \& Transducers Journal, vol. 9, pp. 171-181, 2010.

[7] ICNIRP, "Guideline for limiting exposure to time-varying electric and magnetic fields (1 Hz to $100 \mathrm{kHz}$ )," Health phys, 99(6), pp. 818-836, 2010.

[8] F. W. Grover, Inductance Calculations: Dover Phenix Editions, 2004.

[9] K. Tashiro, A. Matsuoka, and H. Wakiwaka, "Simple-Box-9 coil system: A novel approach to design of a square coil system for producing uniform magnetic fields," Materials Science Forum, vol. 670, pp. 275-283, 2011.

[10] A. Matsuoka, K. Matsumura, A. Kubota, K. Tashiro, and H. Wakiwaka, "Residual magnetization measurements of a motor to be used in satellites," Proc. of SPIE, vol. 7500, pp. 750012-1 - 750012-6, 2009.

[11] A. Kurs, A. Karalis, R. Moffatt, J. D. Joannopoulos, P. Fisher, and M. Soljacic, "Wireless power transfer via strongly coupled magnetic resonances," Science, vol. 317, pp. 83-86, 2007.

TABLE I

SPECIFICATIONS OF ENERGY HARVESTING MODULES

\begin{tabular}{lcccc}
\hline \hline Name of harvesting module & Coil1 & Coil2 & Coil3 & Coil4 \\
\hline Coil width, $c(\mathrm{~mm})$ & 50.0 & 70.0 & 50.0 & 70.0 \\
Inner diameter, $2 c(\mathrm{~mm})$ & 100 & 140 & 100 & 140 \\
Outer diameter, $4 c(\mathrm{~mm})$ & 200 & 280 & 200 & 280 \\
Volume, $4 \pi c^{3}\left(10^{3} \mathrm{~mm}^{3}\right.$ or cm $\left.^{3}\right)$ & 1570 & 4310 & 1570 & 4310 \\
Spacing factor, $\beta$ & 0.850 & 0.938 & 0.108 & 0.107 \\
Number of windings, $n$ & 8500 & 18395 & 1080 & 2104 \\
Inductance, $L(\mathrm{H})$ & 9.04 & 60.6 & 0.15 & 0.77 \\
Resistance, $R(\Omega)$ & 345 & 1060 & 44.2 & 117 \\
Resonant capacitor, $C(\mu \mathrm{F})$ & 0.7 & 0.11 & 47 & 8.6 \\
Load, $R(\Omega)$ & 320 & 1000 & 45 & 120 \\
Resonance frequency, $f_{0}(\mathrm{~Hz})$ & 62.7 & 61.8 & 59.9 & 61.8 \\
Quality factor,$Q$ & 5.25 & 11.0 & 0.64 & 1.28 \\
Half band width ${ }^{\mathrm{b}}, f_{0} / Q(\mathrm{~Hz})$ & 11.9 & 5.60 & 93.8 & 48.3 \\
\hline \hline
\end{tabular}

TABLE II

PARAMETERS FOR THE MODULE WITH IRON RODS

\begin{tabular}{ccc}
\hline \hline $\begin{array}{c}\text { Number of iron rods }{ }^{a} \\
\text { inserted to "Coill" }\end{array}$ & $\begin{array}{c}\text { Measured inductance, } \\
L(\mathrm{H})\end{array}$ & $\begin{array}{c}\text { Resonant capacitor, } \\
C(\mu \mathrm{F})\end{array}$ \\
\hline 0 & 9.2 & 0.7 \\
1 & 10.5 & 0.67 \\
2 & 11.5 & 0.57 \\
3 & 12.2 & 0.4733 \\
4 & 13.1 & 0.47 \\
7 & 15.0 & 0.47 \\
\hline \hline
\end{tabular}

${ }^{\mathrm{a}}$ The diameter and length were $25 \mathrm{~mm}$ and $105 \mathrm{~mm}$.

TABLE III

COMPARISON OF ENERGY MODULES

\begin{tabular}{|c|c|}
\hline & Power density $\left(\mu \mathrm{W} / \mathrm{cm}^{3}\right)$ \\
\hline Solar (outdoors, direct sun) & 15,000 \\
\hline Solar (outdoors, cloudy, day) & 150 \\
\hline Solar (indoors, office desk) & 6 \\
\hline Vibrations(piezoelectric conversion) & 250 \\
\hline Vibrations(electrostatic conversion) & 50 \\
\hline Acoustic noise & 0.0003 at $75 \mathrm{~dB}, 0.96$ at $100 \mathrm{~dB}$ \\
\hline Temperature gradient & 15 at $10^{\circ} \mathrm{C}$ \\
\hline Shoe inserts & 330 \\
\hline Batteries (non-rechargeable lithium) & 45 \\
\hline Batteries (rechargeable lithium) & 7 \\
\hline Hydrocarbon fuel (micro heat engine) & 333 \\
\hline Fuel cells (methanol) & 280 \\
\hline Power line noise (Coil1, air-core) & 0.81 at $21.2 \mu \mathrm{T}$, 71 at $200 \mu \mathrm{T}^{\mathrm{b}}$ \\
\hline Power line noise (Coil2, air-core) & 1.47 at $21.2 \mu \mathrm{T}^{\mathrm{a}}, 130$ at $200 \mu \mathrm{T}^{\mathrm{b}}$ \\
\hline Power line noise (Coil3, air-core) & 0.08 at $21.2 \mu \mathrm{T}^{\mathrm{a}}, 7.6$ at $200 \mu \mathrm{T}^{\mathrm{b}}$ \\
\hline Power line noise (Coil4, air-core) & 0.21 at $21.2 \mu \mathrm{T}^{\mathrm{a}}, 18$ at $200 \mu \mathrm{T}^{\mathrm{b}}$ \\
\hline $\begin{array}{l}\text { The top and middle part of the } \\
\text { group[1]. The bottom part of the tab } \\
\text { power density was estimated for one ye } \\
{ }^{\mathrm{a}} \text { Measured value } \\
{ }^{\mathrm{b}} \text { Estimated value }\end{array}$ & $\begin{array}{l}\text { were summarized by Roundy } \\
\text { as summary of our results. The } \\
\text { fetime. }\end{array}$ \\
\hline
\end{tabular}




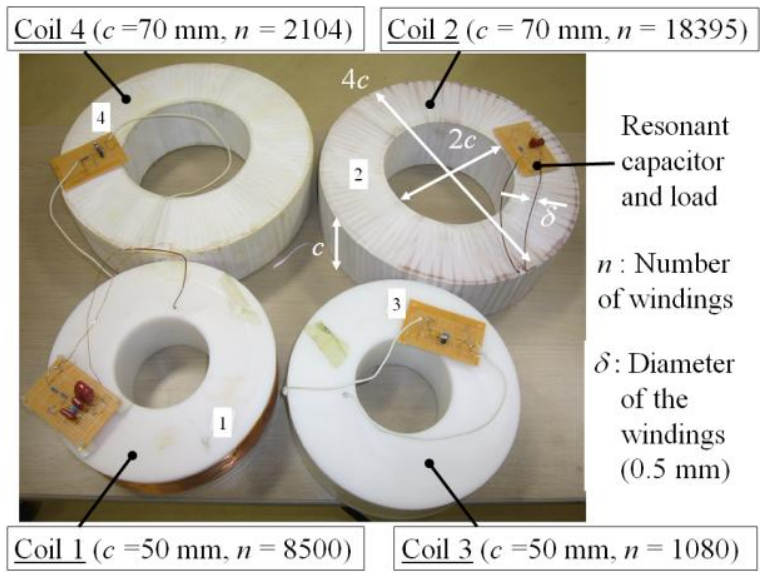

Fig. 1. Our developed energy harvesting modules.

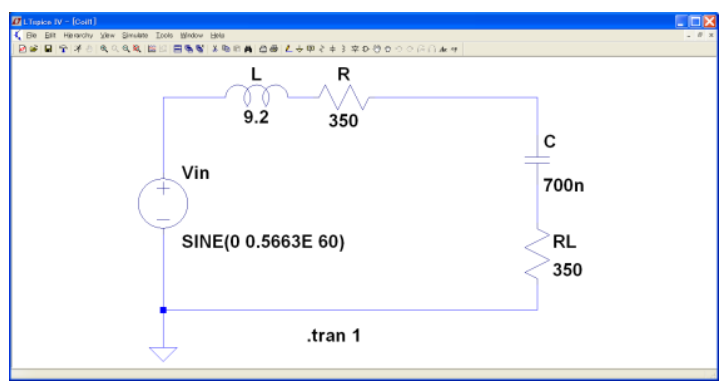

Fig. 2. A model of an energy harvesting module for a circuit simulator.

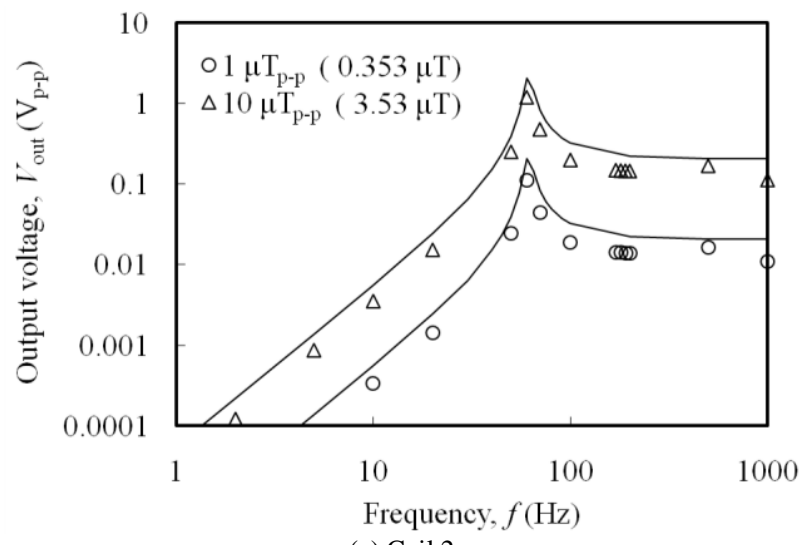

(a) Coil 2

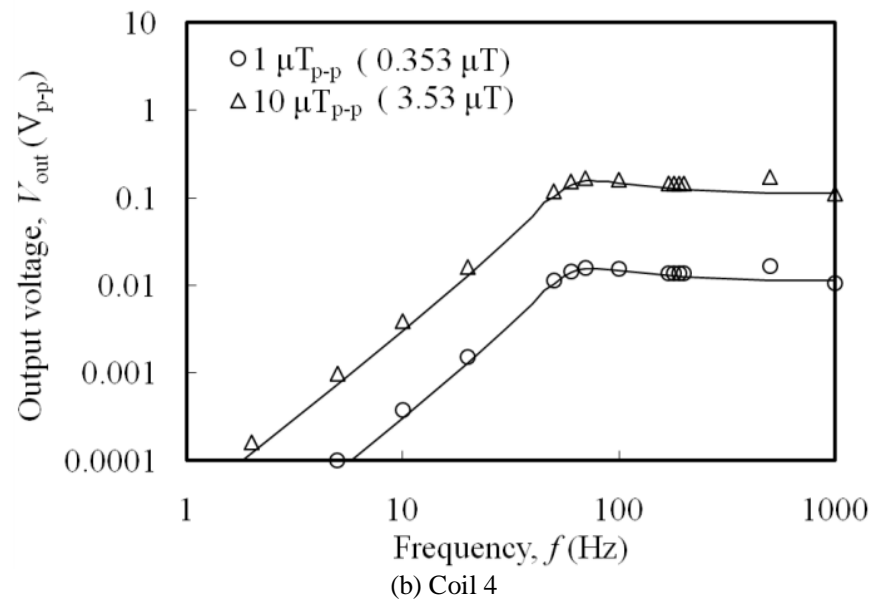

Fig. 3. Examples of the frequency dependency of the modules, Coil2 and Coil4. Plots represent the measured results, and lines represent the simulated results.

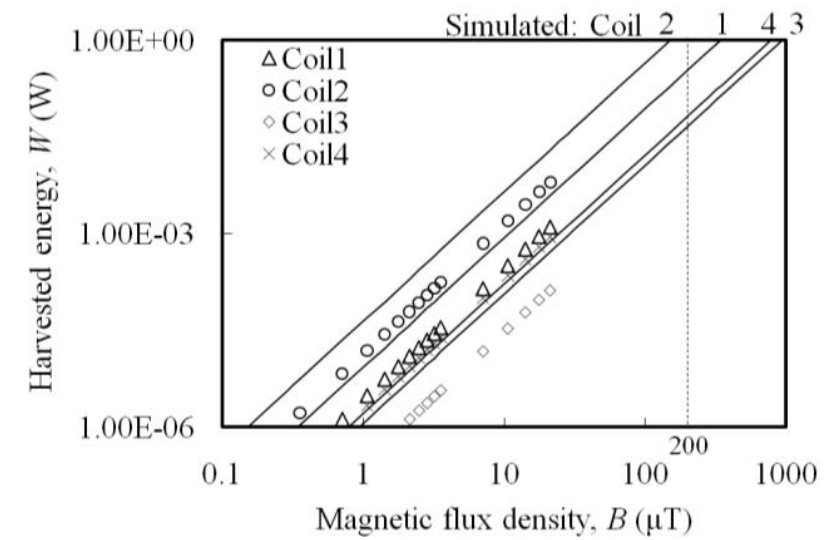

Fig. 4. Amplitude dependency of the modules. Plots represent the measured results, and lines represent the simulated results.

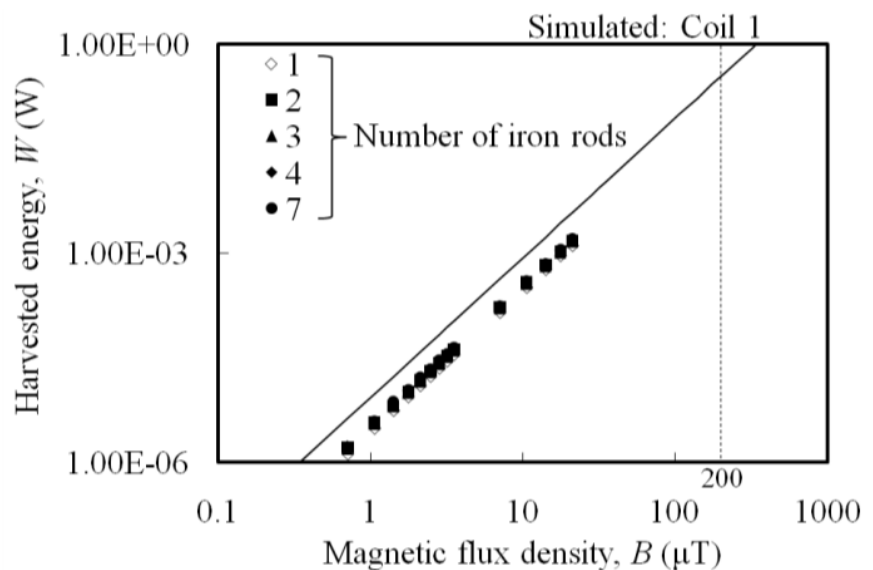

Fig. 5. Amplitude dependency of the module, Coil1, with iron rods. Plots represent the measured results, and lines represent the simulated results of the module without magnetic material.

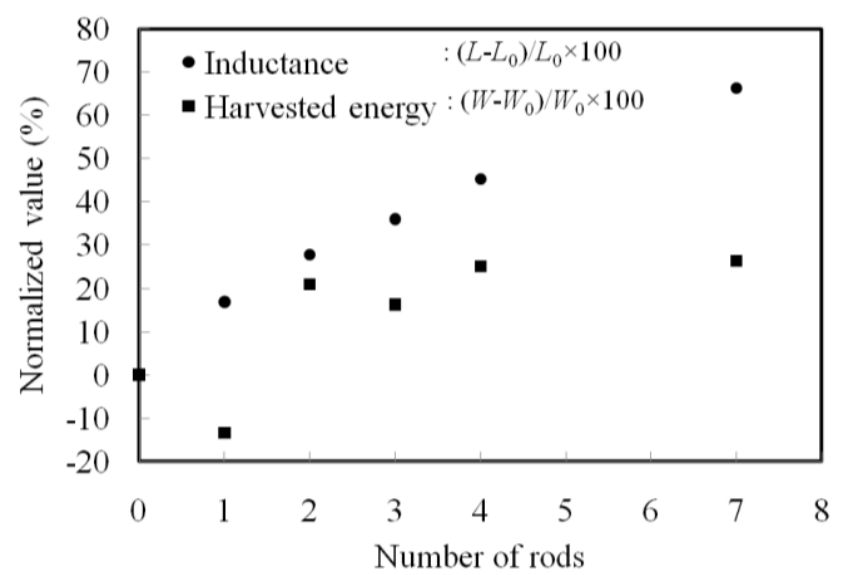

Fig. 6. Comparison of increasing rates of the inductance and harvested energy. Where, $L_{0}(\mathrm{H})$ and $L(\mathrm{H})$ represent the inductance of the coil, Coill, without or with iron cores, respectively. $W_{0}(\mathrm{~W})$ and $W(\mathrm{~W})$ represent the harvested energy from a magnetic field of $21.2 \mu \mathrm{T}$ at $60 \mathrm{~Hz}$ with the module, Coill, without or with iron cores. 